\title{
Deuda Externa, Crecimiento y Sostenibilidad
}

\author{
Dívida externa, crescimento e sustentabilidade
}

RESUMO: O artigo apresenta um modelo destinado a definir e discutir a sustentabilidade das dívidas externas em "mercados emergentes". A primeira condição de sustentabilidade é a existência de um máximo na relação dívida-produto. Com algumas hipóteses de comportamento simples, mostra-se que a sustentabilidade depende do índice inicial de dívida-exportação, da taxa de crescimento das exportações e do prêmio de risco-país. Um prêmio de risco país endógeno dá espaço para múltiplos equilíbrios. O modelo permite a discussão da vulnerabilidade frente aos choques financeiros e a propensão da economia a pular para caminhos insustentáveis. A primeira condição de sustentabilidade não é rigorosa. Duas condições adicionais de sustentabilidade são adicionadas: uma taxa de crescimento positiva e um mínimo no índice interno de absorção / saída. A discussão sobre o equilíbrio múltiplo, a vulnerabilidade e a potencial instabilidade da sustentabilidade é então ampliada.

PALAVRAS-CHAVE: Sustentabilidade; dívida externa; crescimento.

ABSTRACT: The paper presents a model intended to define and discuss the sustainability of external debts in "emergent markets". The first sustainability condition is the existence of a maximum in the debt-output ratio. With some simple behavior hypotheses, it is shown that sustainability depends on the initial debt-exports ratio, the rate of growth of exports and the country-risk premium. An endogenous country-risk premium gives room for multiple equilibrium. The model allows the discussion of vulnerability vis-à-vis financial shocks and the propensity of the economy to jump to unsustainable paths. The first sustainability condition is not a stringent one. Two additional sustainability conditions are added: a positive rate of growth and a minimum in the domestic absorption-output ratio. The discussion of multiple equilibrium, vulnerability and the potential instability of sustainability is then extended. KEYWORDS: Sustainability; external debt; growth. JEL Classification: F34; F32.

\footnotetext{
* Investigador titular del Centro de Estudios de Estado y Sociedad - CEDES, Buenos Aires, Argentina. E-mail: frenkelr@bapro.com.ar. Submetido: agosto 2003; aceito: setembro 2003.

* El autor agradece los comentarios a una versión preliminar de José Antonio Ocampo, Lance Taylor y Sebastián Katz. Martín Rapetti ayudó en la realización de simulaciones del modelo. Aunque poco de esos ejercicios quedó reflejado en la versión aquí pre- sentada, su colaboración fue importante para el resultado final.
} 


\section{INTRODUCCIÓN}

En 2001 la Revista de la CEPAL publicó un artículo de mi autoría ${ }^{1}$ en el que se discute la situación de los países de América Latina en el contexto de globalización financiera de fines de la década de los años noventa. Ese trabajo toca temas de deuda y financiamiento externo, haciendo referencia, entre otras cuestiones, al riesgo país, a la sostenibilidad de la deuda y a la existencia de equilibrios múltiples. En el presente artículo hacemos una presentación analítica de los temas tratados menos formalmente en el trabajo mencionado.

El artículo examina la dinámica de la deuda externa desde el punto de vista del balance de pagos y utilizando el concepto de sostenibilidad. ${ }^{2}$

La sostenibilidad de la deuda externa implica que no cabe esperar grandes dificultades para que los contratos suscritos se cumplan en tiempo y forma. Obviamente, esto no garantiza el cumplimiento de esos contratos. La sostenibilidad es un juicio sobre hechos futuros inciertos, basado en información presente y conjeturas probables. Las condiciones de sostenibilidad que discutimos en este trabajo son razonables a priori.

La tasa de interés que confronta el país, determinada por la tasa internacional y la prima de riesgo país, juega un rol importante en nuestro análisis. El modelo que presentamos refleja algunos rasgos estilizados de las economías de los "mercados emergentes”. Estas son las economías en desarrollo que estuvieron involucradas en el proceso de globalización financiera en tres aspectos: abriendo e integrando el mercado financiero local con el internacional, absorbiendo flujos netos de capital y acumulando consecuentemente un stock significativo de obligaciones externas. Suponemos que los países que consideramos han emitido cierto monto de deuda soberana en moneda internacional (dólares) que es comerciada en el mercado financiero internacional y en el mercado local. La información sobre los precios de los bonos es libre y cualquier agente local o extranjero puede adquirir los bonos soberanos a precios de mercado.

\section{INTEGRACIÓN SEGMENTADA: LA TASA REAL DE INTERÉS Y LA PRIMA DE RIESGO PAÍS 3}

Hay significativas diferencias entre las tasas de interés de las economías desarrolladas y las economías de los mercados emergentes, pese a la integración que existe entre los mercados financieros. En este punto consideramos la determinación de la tasa de interés en el mercado emergente.

\footnotetext{
${ }^{1}$ Frenkel, Roberto (2001).

${ }^{2}$ Ver al respecto Simonsen (1985) y Bhaduri (2001).

${ }^{3}$ Una discusión de la “integración segmentada” se encuentra en Frenkel (2001).
} 
Como fue mencionado, el país emitió bonos soberanos en dólares que son transados en mercados secundarios. La cotización de estos bonos determina su rendimiento, que denominamos $i$. Convencionalmente, este rendimiento puede ser descompuesto en dos términos:

$$
i=r^{*}+k(1)
$$

donde $r^{*}$ es el rendimiento del bono soberano de Estados Unidos del mismo plazo y características y $k$ es la prima de riesgo país.

Señalamos en primer lugar que $i$ es el costo de oportunidad de cualquier inversión extranjera en el país, en la medida que el activo en cuestión, real o financiero, es sujeto de riesgo soberano. En consecuencia, el costo del crédito internacional recibido por los bancos locales u otros agentes privados debería ser generalmente mayor o igual a $i$.

Para facilitar la presentación de la determinación de la tasa de interés real, pero sin perder generalidad, suponemos que el sistema financiero doméstico está parcialmente dolarizado. Esto significa que además de depósitos y créditos en pesos los bancos también toman y otorgan depósitos y créditos denominados en dólares.

Como los bancos locales pueden adquirir bonos soberanos que rinden $i$, esta tasa es el costo de oportunidad de los créditos bancarios denominados en dólares. En consecuencia, $i$ es el piso del precio de los préstamos bancarios en dólares, aunque el crédito no haya sido fondeado en el mercado internacional. La tasa de interés de los préstamos en dólares debe ser mayor o igual a $i$. Suponemos que es igual a $i$.

Para determinar la tasa de interés en pesos debe tenerse en cuenta la tendencia esperada del tipo de cambio:

$$
j=i+E(e)+\pi \quad(2) \quad \pi>0
$$

donde $j$ es la tasa de interés nominal en pesos, $E(e)$ es la tasa esperada de devaluación nominal y $\pi$ representa la prima de riesgo cambiario.

Sean $p$ y $p *$ las tasas de inflación local e internacional, respectivamente. Podemos restar $p$ de ambos miembros de (2) y sumar y restar $p^{*}$ en el segundo miembro:

$$
j-p=\left(i-p^{*}\right)+\left[E(e)-p+p^{*}\right]+\pi
$$

Resulta así una expresión donde $j$ - $p$ es la tasa de interés real en pesos y $E(e)$ $p+p^{*}$ es la tasa esperada de devaluación real. Con esta expresión es fácil ver que la tasa de interés real en pesos debe ser mayor o igual a $i-p^{*}$, salvo en el caso de una tendencia esperada de apreciación real suficientemente importante como para compensar el riesgo cambiario $\pi$. No tomaremos en consideración este caso. Consecuentemente, asumimos:

$$
j-p i-p^{*}
$$

Para simplificar, suponemos $p^{*}=0$ (lo que es equivalente a considerar $p$ como el diferencial entre la inflación local y la internacional) y suponemos también que (4) se verifica como igualdad:

$$
r=j-p=i=r^{*}+k
$$




\section{EL FINANCIAMIENTO EXTERNO}

El país ha recibido ingresos de capital que acumulan un monto $D(t)$ de deuda externa. Los ingresos de capital resultan de la colocación de bonos de plazo $L$, que rinden la tasa de interés internacional $r^{*} y$ que pagan el total del capital al vencimiento.

El balance en cuenta corriente (CC) es:

$C C=X-M-r * D$

donde $X$ son las exportaciones, $M$ las importaciones y $r^{*} D$ son los intereses devengados por la deuda externa.

Consideramos que no puede haber variación de reservas y suponemos (por el momento y a los fines de la presentación del modelo) que la cuenta corriente es deficitaria. La necesidad de financiamiento $(N F)$ es:

$N F=-C C+\lambda D=-X+M+r^{*} D+\lambda . D$

donde es la fracción de la deuda que vence en $t$.

Como los bonos se colocan por debajo de $\left(k \begin{array}{l}k\end{array}\right)$, para obtener un monto de financiamiento $N F$ se debe colocar un volumen $Z$ de nueva deuda tal que:

$Z e^{r^{*} L}=N F e^{\left(r^{*}+k\right) L}$ y por lo tanto

$Z=N F e^{k L}$

La variación de deuda es entonces:

$d D=\frac{\partial D}{\partial t}=Z-\lambda D$

Reemplazando por (6) y (7) y reagrupando se obtiene:

$$
d D=\left[e^{k L} r^{*}+\lambda\left(e^{k L}-1\right)\right] D-e^{k L} X+e^{k L} M
$$

Hacemos:

$$
\begin{aligned}
& \delta=e^{k L}, \delta \geq 1 \quad \text { ya que } k \geq 0 \\
& \gamma=\delta r^{*}+\lambda(\delta-1), \gamma \geq r^{*}>0 \quad \text { ya que } \delta \geq 1
\end{aligned}
$$

y la ecuación (8) se expresa:

$$
d D=\gamma D-\delta(X-M)
$$

La variación de deuda se descompone en un término puramente financiero, que depende del stock de deuda y un término que depende del balance comercial. El primer término representa el costo del financiamiento de la suma de los intereses devengados por la deuda existente más la refinanciación de los vencimientos de capital. Si hay déficit comercial $(\mathrm{X}<\mathrm{M})$, el segundo término representa el costo de su financiamiento. En ambos términos aparece el factor $\partial$ como multiplicador. Este es mayor cuanto mayor es la prima de riesgo país y consecuentemente, es más alta la tasa de interés que debe comprometer el país para obtener financiamiento. 
Obsérvese que la ecuación (9) es válida también en el caso de superávit comercial $(\mathrm{X}>\mathrm{M})$. En este caso el resultado del balance comercial se aplica a la financiación de los intereses y vencimientos de capital de la deuda existente, lo que es equivalente a suponer que el resultado comercial se aplica a la compra de bonos de deuda a precios de mercado. Si el superávit comercial es lo suficientemente grande, el valor absoluto del segundo término puede ser mayor que el primero y el stock de deuda se contrae. Puede verse entonces, que es innecesario el supuesto de déficit de cuenta corriente que hicimos arriba con fines expositivos, ya que la ecuación (9) es válida en cualquier caso. Si la prima de riesgo es nula, la ecuación (9) se reduce a:

$$
d D=r * D-X+M \text {, }
$$

esto es, la variación de deuda es igual al resultado de la cuenta corriente cambiado se signo.

Dividiendo por $D$ la ecuación (9) se obtiene la tasa de crecimiento de la deuda:

$$
\frac{d D}{D}=\hat{D}=\gamma-\delta \frac{X-M}{D}
$$

Sea $Y$ el producto e $y$ su tasa de crecimiento. La tasa de variación de la relación deuda/producto es:

$\frac{d(D / Y)}{D / Y}=\hat{D}-y=\gamma-y-\delta \frac{X-M}{D}$

que multiplicando $y$ dividiendo por $Y$ en el último término puede $\mathrm{e}$

$$
\frac{d(D / Y)}{D / Y}=\hat{D}-y=\gamma-y-\delta\left(\frac{X-M}{Y}\right) / \frac{D}{Y}
$$

La tasa de variación de la relación deuda/producto en la ecuación (12) está expresada en función de relaciones macroeconómicas de consideración habitual. ${ }^{4}$

\section{LAS TASAS DE CRECIMIENTO DEL PRODUCTO Y LAS IMPORTACIONES}

Completamos el modelo haciendo endógenas las tasas del producto $y$ de las importaciones. La tasa de crecimiento de las exportaciones la supondremos exógena.

Incorporamos al modelo la influencia de la prima de riesgo país sobre la econo-

\footnotetext{
${ }^{4}$ Por ejemplo, si $\mathrm{L}=10$ años, $\mathrm{r}^{*}=5 \%$ y la prima de riesgo país $\mathrm{k}=0.03$ (300 pb), entonces resulta $\gamma=$ 0.1025 . Con equilibrio comercial, la tasa de variación de la relación deuda/producto se hace cero solo si el producto crece al $10.25 \%$ anual. Si la tasa de crecimiento de la economía es $7 \%$ y la relación deuda/ producto es $50 \%(\mathrm{D} / \mathrm{Y}=0.5)$, se requiere un superávit comercial de $1.2 \%$ del producto para estabilizar la relación deuda/producto.
} 
mía real a través de la tasa de interés real. Optamos por la forma más simple, asumiendo que la tasa de crecimiento de la economía es función de la tasa de interés:

$$
y=y(r)=\bar{y}+\rho r=\bar{y}+\rho r^{*}+\rho k, \quad \text { con } \rho<0
$$

donde $\rho$ es la elasticidad producto/tasa de interés.

También hacemos endógena la tasa de crecimiento de las importaciones, asumiendo que ésta es función de la tasa de crecimiento del producto:

$$
m=m(y)=\mu y, \quad \text { con } \mu>0
$$

donde $\mu$ es la elasticidad importaciones/producto.

\section{LA DINÁMICA DE LA DEUDA}

Para resolver la ecuación diferencial (9) y obtener la trayectoria de la deuda $D(t)$ deben especificarse las trayectorias de exportaciones e importaciones:

$$
\begin{aligned}
X & =X_{0} e^{x t} \\
M & =M_{0} e^{m t}
\end{aligned}
$$

donde $x$ y $m$ son las tasas de crecimiento de exportaciones e importaciones, respectivamente. Con esta especificación la ecuación (9) resulta:

$$
d D=\gamma D-\delta\left(X_{0} e^{x t}-M_{0} e^{m t}\right)
$$

En el caso general en el cual $x$ y $m$ la solución de la ecuación diferencial es:

$$
D(t)=\left[D_{0}+\delta\left(\frac{X_{0}}{x-\gamma}-\frac{M_{0}}{m-\gamma}\right)\right] e^{\gamma t}-\delta\left(\frac{X_{0}}{x-\gamma} e^{x t}-\frac{M_{0}}{m-\gamma} e^{m t}\right)
$$

Como es obvio, la evolución de la deuda depende de los parámetros financieros comentados arriba, de las tasas de crecimiento de exportaciones e importaciones y de las condiciones iniciales de superávit o déficit comercial.

Enfocaremos en la relación deuda/producto nuestra discusión del problema de sostenibilidad de la deuda. La trayectoria del producto es:

$$
Y=Y_{0} e^{y t}
$$

Consecuentemente, utilizando (16), la relación deuda/producto sigue la trayectoria:

$$
\frac{D}{Y}=\frac{D_{0}}{Y_{0}} e^{(\gamma-y) t}-\frac{X_{0}}{Y_{0}} \frac{\delta}{x-\gamma}\left[e^{(x-y) t}-e^{(\gamma-y) t}\right]+\frac{M_{0}}{Y_{0}} \frac{\delta}{m-\gamma}\left[e^{(m-y) t}-e^{(\gamma-y) t}\right]
$$

La evolución de la relación deuda/producto, como la deuda, también depende de las condiciones iniciales del balance comercial. Queremos eliminar este elemen- 
to para enfocar el análisis sobre los parámetros. Para esto, suponemos en adelante que las condiciones iniciales son de equilibrio comercial. Por lo tanto, hacemos

$$
\frac{D}{Y}=\frac{X_{0}}{Y_{0}}\left[e^{(\gamma-y) t}\left(\frac{D_{0}}{X_{0}}+\frac{\delta}{x-\gamma}-\frac{\delta}{m-\gamma}\right)-\left(\frac{\delta}{x-\gamma} e^{(x-y) t}-\frac{\delta}{m-\gamma} e^{(m-y) t}\right)\right]
$$

La expresión (18) pone de manifiesto las condiciones iniciales que influyen en la evolución de la relación deuda/producto: el grado de apertura de la economía ( $X$ $\left.{ }_{0} / Y_{0}\right)$ y la relación deuda/exportaciones $\left(D_{0} / X_{0}\right)$. Pero la primera aparece como un factor de escala que no influye en las características de la trayectoria de la relación deuda/producto. En cambio, esta trayectoria depende esencialmente de la relación inicial deuda/exportaciones. Una devaluación, por ejemplo, a través del aumento del precio relativo de los bienes transables, aumenta el grado de apertura de la economía y consecuentemente multiplica por este factor toda la curva de la relación deuda/producto. Pero la relación deuda/exportaciones permanece inalterada por la devaluación y por lo tanto ésta no modifica la forma y características de la trayectoria de la relación deuda/producto. La devaluación solo incide en la forma de esta curva por sus efectos reales, a través de sus efectos sobre las tasas de las exportaciones e importaciones.

Con la introducción de los supuestos para la economía real (13) y (14) queda definido un modelo cuyo funcionamiento puede explicarse observando la ecuación (18). El aumento de la prima de riesgo país tiende a elevar la relación deuda/producto por el lado financiero, porque aumentan los parámetros financieros $\delta$ y $\gamma$. La incorporación del efecto recesivo del aumento de la tasa de interés agrega otro elemento por el lado real: el aumento de la prima de riesgo tiende a reducir la tasa de crecimiento del producto y consecuentemente tiende por su lado también a elevar la relación deuda/producto. Pero la reducción de la tasa de crecimiento de la economía induce una caída en el ritmo de crecimiento de las importaciones. Con la tasa de crecimiento de las exportaciones inalterada, el superávit comercial se incrementa (o el déficit se reduce) y este efecto tiende a disminuir la relación deuda/ producto.

\section{DÉFICIT FINANCIERO Y SUPERÁVIT COMERCIAL}

Para analizar la dinámica de la relación deuda/producto establecemos algunas restricciones sobre los parámetros del modelo. La mejor manera de presentarlas es a partir de ecuación (11), reescrita con el supuesto de condiciones iniciales de equilibrio comercial $\left(M_{0}=X_{0}\right)$.

$$
\hat{D}-y=\gamma(k)-y(k)-\delta(k) X_{0} \frac{e^{x t}-e^{m(k) t}}{D}
$$

La ecuación (19) expresa la tasa de crecimiento de la relación deuda/producto. Hemos explicitado en ella los parámetros que dependen de la prima de riesgo país. 
Imaginemos que en $t<0$ la economía afrontaba cierta prima de riesgo país (llamémosla $\left.k_{0}\right)$ tal que la tasa de crecimiento $\left(y_{0}\right)$ resultaba igual al costo de refinanciar los intereses y los vencimientos de capital de la deuda externa $\left(\gamma_{0}\right)$. Con esa tasa de crecimiento de la economía, la tasa de aumento de las importaciones $\left(m_{0}\right)$ igualaba la tasa de aumento de las exportaciones $\left(x_{0}\right)$, de modo que la economía crecía con equilibrio comercial. En $t<0$ la ecuación (19) resultaba:

$$
\hat{D}-y=\gamma_{0}-y_{0}=0
$$

La tasa de crecimiento de la relación deuda/producto era nula, la economía se encontraba sobre una trayectoria de deuda sostenible, con una relación constante deuda/producto.

Imaginemos ahora que por un efecto de contagio la prima de riesgo país aumenta en $t \geq 0\left(k>k_{0}\right)$. Consecuentemente, $g$ aumenta y se reduce al mismo tiempo la tasa de crecimiento $y$ ( $\left.\mathrm{g}>\mathrm{g}_{0}, y<y_{0}\right)$ de modo que $\mathrm{g}>y$. La tasa de crecimiento de la relación deuda/producto se haría positiva por este efecto. Pero la reducción de la tasa de crecimiento induce una caída de la tasa de crecimiento de las importaciones $\left(m<m_{0}\right)$ y consecuentemente $x>m$ en $t \geq 0$. Por otro lado, obsérvese con la ecuación (19) que en $t=0$ la tasa de crecimiento de la relación deuda/producto es positiva:

$$
\hat{D}-y=\gamma_{0}-y_{0}=0
$$

El efecto impacto del aumento de la prima de riesgo es una tendencia creciente de la relación deuda/producto. Pero desde un valor nulo en $t=0$, el superávit comienza a crecer en $t>0$ ¿Bajo qué condiciones el superávit comercial creciente puede compensar el efecto financiero del aumento de la prima de riesgo y hacer sostenible la deuda externa?

En términos generales, el problema planteado establece las siguientes restricciones sobre los parámetros:

$\gamma>y ; x>m$

Con estos supuestos analizamos la dinámica de la relación deuda/producto.

Más allá del problema que los justifica, los supuestos que asumimos parecen ser los únicos interesantes para la discusión de la sostenibilidad de la deuda. Si $\gamma>$ y y la economía no genera superávit comercial, la tendencia de la relación deuda/ producto es explosiva y la deuda es indiscutiblemente insostenible. Por otro lado, si podría discutirse, por ejemplo, cuál es el déficit comercial que tolera la dinámica de la deuda sin hacerse explosiva, pero no parece un tema muy interesante.

\section{PRIMERA CONDICIÓN DE SOSTENIBILIDAD}

La primera condición de sostenibilidad es que la relación deuda/producto no siga una trayectoria explosiva. La curva que expresa la ecuación (16) debe tener un máximo. La condición de primer orden es: 
La condición de segundo orden es:

$$
\begin{aligned}
& \frac{\partial(D / Y)}{\partial t}=\frac{X_{0}}{Y_{0}}\left\{(\gamma-y) e^{(\gamma-y) t}\left(\frac{D_{0}}{X_{0}}+\frac{\delta}{x-\gamma}-\frac{\delta}{m-\gamma}\right)-\right. \\
& \left.-\left[\frac{\delta}{x-\gamma}(x-y) e^{(x-y) t}-\frac{\delta}{m \gamma}(m-y) e^{(m-y) t}\right]\right\}=0
\end{aligned}
$$

La condición de segunda orden es:

$$
\begin{aligned}
& \frac{\partial^{2}(D / Y)}{\partial t^{2}}=\frac{X_{0}}{Y_{0}}\left\{(\gamma-y)^{2} e^{(\gamma-y) t}\left(\frac{D_{0}}{X_{0}}+\frac{\delta}{x-\gamma}-\frac{\delta}{m-\gamma}\right)-\right. \\
& \left.-\left[\frac{\delta}{x-\gamma}(x-y)^{2} e^{(x-y) t}-\frac{\delta}{m-\gamma}(m-y)^{2} e^{(m-y) t}\right]\right\}<0
\end{aligned}
$$

Con la expresión (20) resulta claro que entre las condiciones iniciales, la existencia de un máximo depende solamente de la relación deuda/exportaciones. Como $X_{0} / Y_{0}>0$, la expresión entre corchetes debe anularse para que se verifique (20), y ésta depende exclusivamente de la condición inicial.

La existencia de un máximo (de un valor $t>0$ que satisface (20)) depende, en primer lugar, de la tasa de crecimiento de las exportaciones. Si, existe un máximo siempre y la deuda es sostenible, cualquiera sean los valores de las tasas de crecimiento del producto y las importaciones (con la restricciones ya formuladas $\gamma>y$; $x>m$ ). La demostración se presenta en el Anexo. En términos del problema planteado arriba, es el caso de una economía que experimenta un shock sobre la prima de riesgo tal que $\gamma>y_{0}>y$, pero el valor de $\gamma$ resulta todavía inferior a la tasa de

La verificación de la condición de segundo orden es en realidad redundante porque sabemos que

, de modo que si (20) tiene un punto de inflexión, debe ser un máximo.

crecimiento de las exportaciones $\left(\gamma<x_{0}\right)$. En este caso la relación deuda/producto tiende a crecer a consecuencia del shock, pero alcanza un máximo.

Si $x<\gamma$, la condición para que se verifiquen (20) y (21) en $t>0$ es:

$$
\frac{\delta(x-m)}{(x-\gamma)(m-\gamma)}>\frac{D_{0}}{X_{0}}
$$

La demostración se presenta en el Anexo. En términos del problema planteado, es el caso de un shock sobre la prima de riesgo tal que el nuevo valor de $\gamma$ supera la tasa de crecimiento de las exportaciones. En este caso, la deuda es sostenible solo si se verifica (22). En caso contrario la relación deuda/producto crece explo-

\footnotetext{
${ }^{5}$ La verificación de la condición de segundo orden es en realidad redundante porque sabemos que $\frac{\partial(D / Y)}{\partial t}(0)>0$, de modo que si (20) tiene un punto de inflexión, debe ser un máximo.
} 
sivamente. Dados $L$ y $r^{*}$, la tasa de crecimiento de las exportaciones $x$ y las elasticidades $\rho$ y $\mu$, el miembro izquierdo de (22) es una función decreciente de la prima de riesgo país:

$$
H(k)=\frac{\delta(k)[x-m(k)]}{[x-\gamma(k)][m(k)-\gamma(k)]}
$$

La curva $H$ está ilustrada en el panel izquierdo del Gráfico 1. La relación deuda/producto solo tiene un máximo si el valor de $H$ correspondiente a determinado $k$ es mayor que $D_{0} / X_{0}$. La intersección de la curva $H$ con la recta $D_{0} / X_{0}$ determina la prima de riesgo crítica $k_{c}$ tal que la relación deuda/ producto se hace explosiva para todo $k \geq k_{c}$.

Los dos paneles del gráfico ilustran el problema planteado arriba en el caso de que el shock determina un valor. En $t<0$ la prima de riesgo país era $k_{0}$ y el crecimiento de la economía igualaba la tasa de crecimiento de la deuda. Si la prima de

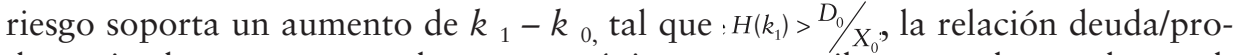
ducto tiende a crecer, pero alcanza un máximo, como se ilustra en el segundo panel. En cambio, si el aumento de la prima de riesgo es $k_{2}-k_{0}$, de magnitud suficiente para que, la relación deuda/producto tiende a crecer en forma explosiva, como se ilustra también en el segundo panel.

Podemos ahora resumir las conclusiones de este punto, expresándolas en términos del problema planteado arriba. Frente a un aumento de la prima de riesgo, la sostenibilidad de la deuda depende en primer lugar de la tasa de crecimiento de las exportaciones. Si la economía venía creciendo con equilibrio comercial, pero con apertura comercial en aumento, la deuda es sostenible si, después del shock, $\gamma$ sigue siendo inferior a la tasa de crecimiento de las exportaciones. En otro caso (por ejemplo, si la economía venía creciendo con coeficientes constantes de comercio exterior/ producto) la sostenibilidad de la deuda depende de la relación deuda/exportaciones. Cuanto menor es la relación deuda/exportaciones, mayor es el aumento de la prima de riesgo que soporta la relación deuda/producto sin tornarse explosiva.

Consideremos ahora dos países cuyas condiciones iniciales y parámetros de comportamiento son iguales, salvo con respecto a sus relaciones de apertura comercial y consecuentemente deuda/exportaciones. Ambos tienen la misma relación inicial deuda/producto, pero la primera economía tiene un coeficiente exportaciones/producto (y consecuentemente importaciones/producto) significativamente mayor que la segunda.

De modo que la primera ostenta una relación deuda/exportaciones significativamente inferior a la segunda. Llamamos Chilmex a la primera economía y Brasarg a la segunda. Ambas economías tienen la misma curva, como se muestra en el Gráfico 2. Supongamos que las dos economías soportan idéntico shock en la prima de riesgo país (tal que el nuevo valor de supera la tasa de crecimiento de las exportaciones), causado, por ejemplo, por una caída generalizada del precio de los bonos de los 
mercados emergentes. Con la nueva prima de riesgo país, la relación deuda/producto crece explosivamente en Brasarg, mientras que la deuda de Chilmex es sostenible. ${ }^{6}$

Gráfico 1
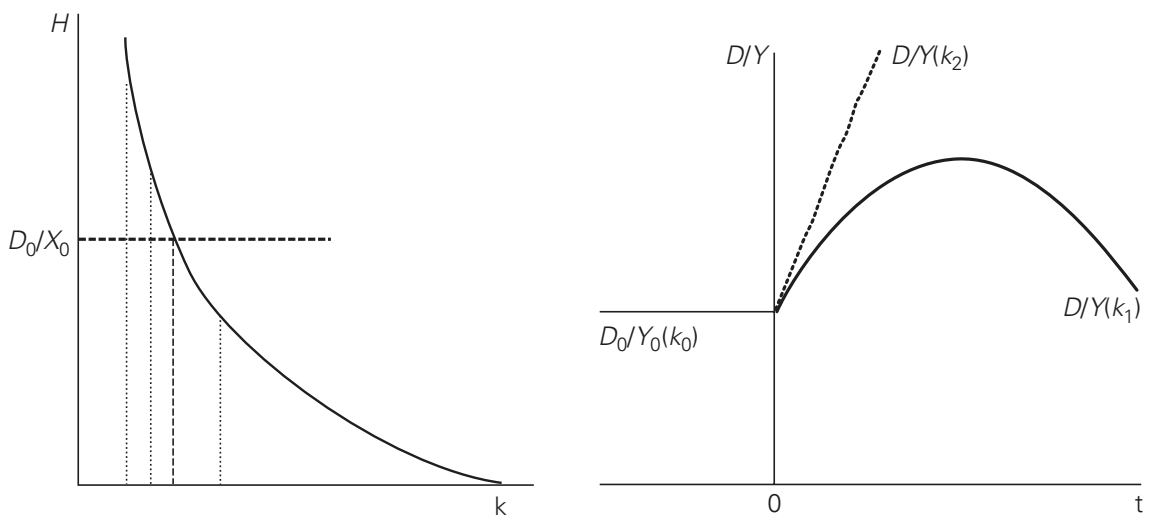

Gráfico 2

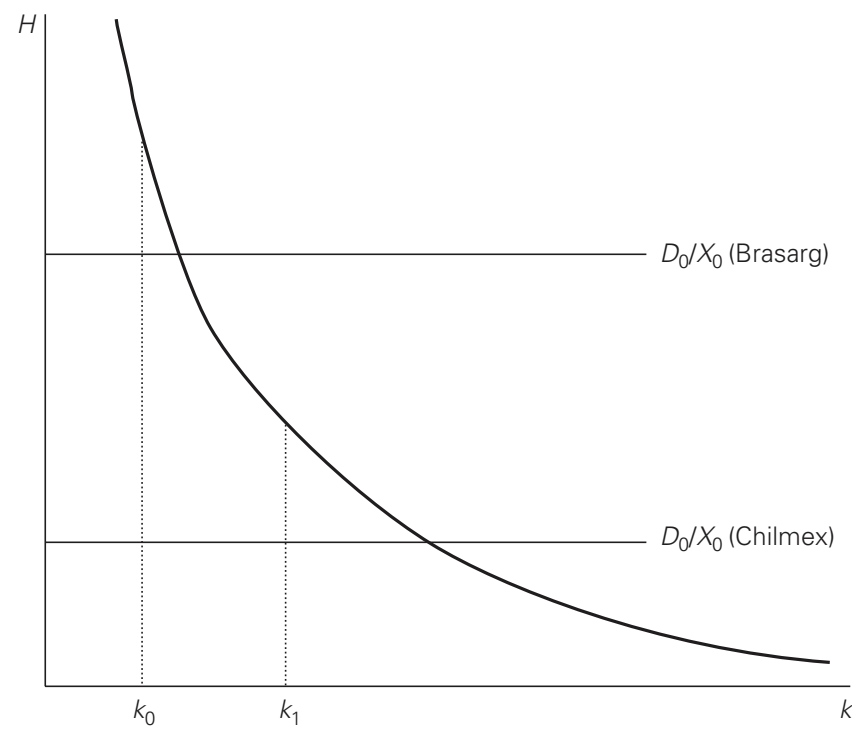

${ }^{6}$ Consideremos un ejemplo numérico. Supongamos que Chilmex y Brasarg tienen la misma relación deuda/ producto $\mathrm{D}_{0} / \mathrm{Y}_{0}=50 \%$. Supongamos también que Chilmex tiene una relación exportaciones/ producto de $33 \%$, mientras que esa relación es de $10 \%$ en el caso de Brasarg. De modo que la relación deuda/ exportaciones es D0/X0 = 1.5 en el caso de Chilmex, y en el caso de Brasarg es D0/X0 = 5. Supongamos para ambas economías $\mathrm{r}^{*}=5 \% ; \mathrm{k} 0=200 \mathrm{pb} ; \mathrm{y} 0=8 \% ; \mathrm{x} 0=10 \% ; \mathrm{m} 0=10 \% ; \rho=-1 \mathrm{y}$ $\mu=1.25$. Estas condiciones, como puede verse, representan una performance excepcionalmente favorable. Con estos parámetros, la deuda de Brasarg se hace insostenible para primas de riesgo por arriba de 800 pb, mientras que la deuda de Chilmex es sostenible hasta primas de riesgo de $2000 \mathrm{pb}$. 


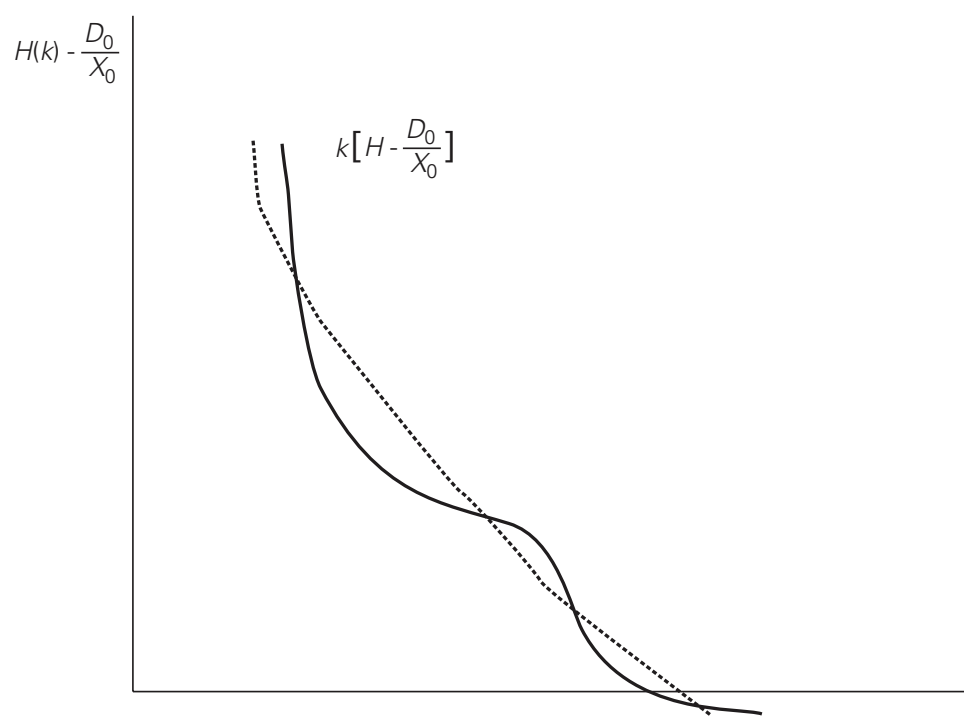

\section{EQUILIBRIOS MÚLTIPLES Y PROFECÍAS AUTOCUMPLIDAS}

Imaginemos que los inversores internacionales incluyen la relación deuda/exportaciones en su evaluación del riesgo país. Ceteris paribus, la prima de riesgo país es mayor cuanto más alta es la relación deuda/exportaciones, porque mayor es la posibilidad de que el país con una relación deuda/exportaciones relativamente alta ingrese en una trayectoria explosiva de deuda frente a una caída del precio de sus bonos. El razonamiento que justificaría esta valoración del riesgo puede tomar en cuenta la reacción de la política económica. Frente a un cambio en las condiciones financieras internacionales que amenaza la sostenibilidad de la deuda externa, el país puede instrumentar medidas para aumentar la tasa de crecimiento de sus exportaciones y garantizar la sostenibilidad. Sin embargo, como puede verse con la ecuación (22), la magnitud del incremento de exportaciones necesario es mayor cuanto mayor es la relación deuda/exportaciones.

El modelo compuesto por la condición de sostenibilidad y la función de prima de riesgo:

$$
k=k\left[H(k)-D_{0} / X_{0}\right] \quad k^{\prime}<0
$$

puede tener equilibrios múltiples, como se ilustra en el Gráfico 3. En el gráfico, las curvas $H(k)$ y $k\left(H, D_{0} / X_{0}\right)$ se cortan en varios puntos. Esto es, hay distintos valores de la prima de riesgo que satisfacen la función y determinan ciertas trayectorias de la relación deuda-producto. Ceteris paribus, la deuda es sostenible con 
una prima de riesgo país pequeña. En cambio, la deuda es insostenible para primas de riesgo tales que

$$
H(k) \leq D_{0} / X_{0}
$$

En el Gráfico 4 ilustramos con Chilmex y Brasarg el modelo en el cual países semejantes pero con diferentes ratios deuda externa/exportaciones enfrentan distintas primas de riesgo país. En el gráfico, la prima de Chilmex $k_{c b}$ es menor que la prima de Brasarg $k_{B}$ porque Brasarg tiene una relación deuda/exportaciones significativamente mayor que Chilmex. Sin embargo, a causa de esta valoración del mercado financiero internacional, la prima de riesgo de Brasarg está próxima a la prima de riesgo crítica que haría insostenible su deuda. En consecuencia, Brasarg es más vulnerable a cambios en las condiciones financieras internacionales que Chilmex, porque un aumento de igual magnitud de las primas de riesgo de ambos países puede hacer insostenible la deuda en Brasarg, mientras que la deuda de Chilmex sigue siendo sostenible.

Por la misma razón, la sostenibilidad de Brasarg es más proclive a profecías autocumplidas. Cuando la prima de riesgo es tal que $H(k)$ tiene un valor próximo a la relación deuda/exportaciones (como es el caso de Brasarg en el Gráfico 4) parece razonable pensar que la conducta de los inversores internacionales sea más proclive a cambios súbitos de opinión, coordinados por señales convencionales internas o internacionales, que conduzcan a aumentos de la prima de riesgo que tornen insostenible la dinámica de la deuda.

\section{CONDICIONES DE SOSTENIBILIDAD COMPLEMENTARIAS}

La existencia de un máximo de la relación deuda/producto no es una condición muy exigente. Además de la condición de no explosividad, la sostenibilidad de la deuda debería satisfacer otras condiciones, como las que discutimos a continuación.

\section{TASA DE CRECIMIENTO POSITIVA}

La primera condición adicional que consideramos es el requisito de que la economía mantenga una tasa de crecimiento positiva.

Frente a un aumento de la prima de riesgo país, puede ocurrir, por ejemplo, que la deuda resulte sostenible de acuerdo al criterio de no explosividad, pero que esa condición se verifique en un contexto de contracción del producto. Si la trayectoria no explosiva está basada en una prolongada contracción del producto y las importaciones los contratos se harán incumplibles en algún momento.

Consecuentemente, este criterio de sostenibilidad es:

$y>0$,

que en términos del problema planteado puede formularse: 


$$
y=y_{0}+\rho\left(k-k_{0}\right)>0
$$

donde $\rho<0$ es la elasticidad tasa de interés del producto y $\left(k-k_{0}\right)$ es el aumento de la prima de riesgo país. De (24) se sigue que:

$$
k-k_{0}<-\frac{y_{0}}{\rho} \quad \text { o también } \quad k<k_{0}-\frac{y_{0}}{\rho}
$$

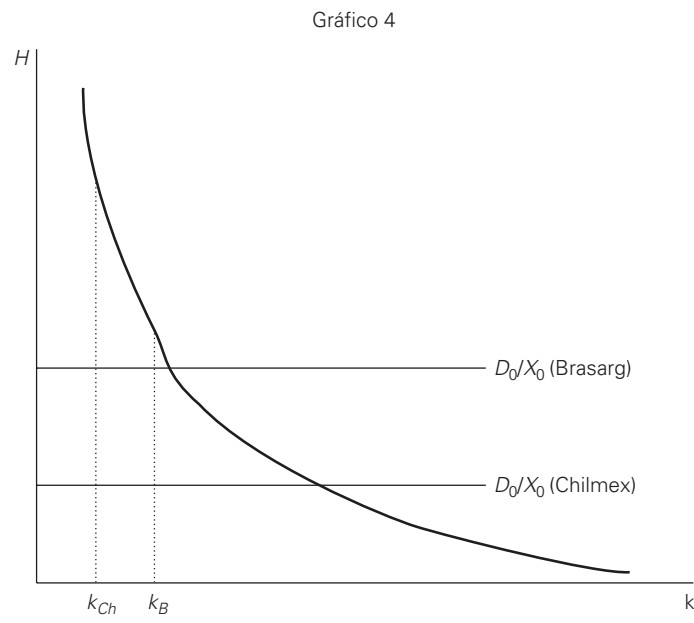

Gráfico 5

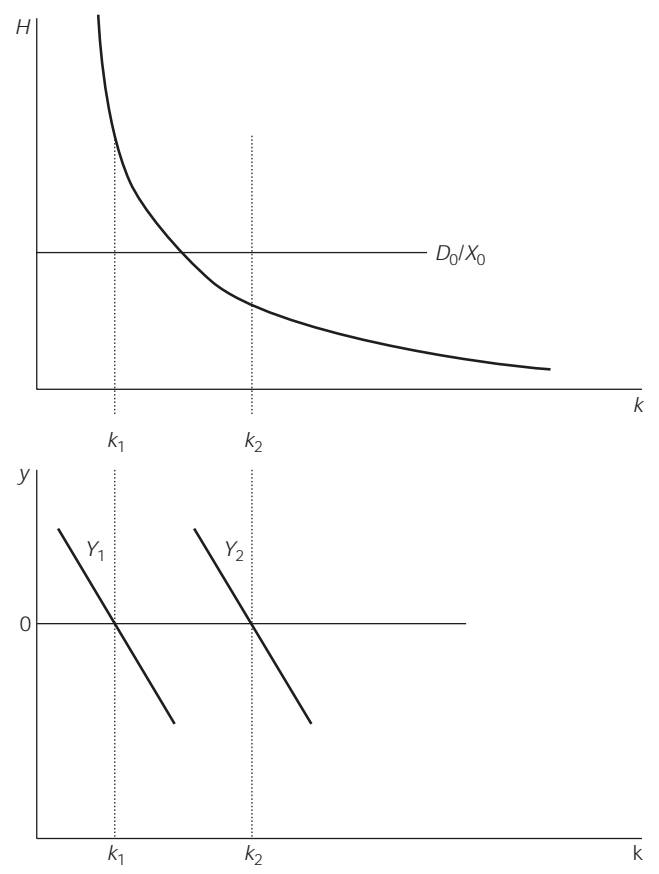


La restricción (25) puede ser operativa para primas de riesgo país inferiores a las que harían insostenible la deuda de acuerdo al criterio de no explosividad. En el Gráfico 5 ilustramos esta posibilidad. En el panel inferior del gráfico dibujamos dos curvas $y\left(r^{*}+k\right)$ que expresan las tasas de crecimiento en función de la tasa de interés. La curva $Y_{2}$ corta la recta $y=0$ con $k=k_{2}$, una prima de riesgo mayor que la que hace explosiva la relación deuda/producto. En este caso, el criterio (25) no es operativo: cuando aumenta la prima de riesgo la deuda se hace explosiva aún con tasas de crecimiento positivas. Por otro lado, si la economía está caracterizada por la curva $Y_{1}$, que corta la recta $y=0$ con una prima de riesgo $k=k_{1}$, inferior a la que haría explosiva la relación deuda/producto, el criterio de sostenibilidad operativo es el que requiere una tasa positiva de crecimiento. En este caso la tasa de crecimiento se hace negativa para primas de riesgo país con las cuales la deuda todavía resultaría sostenible con el criterio de no explosividad.

\section{LÍMITE MÍNIMO DE LA RELACIÓN ABSORCIÓN/PRODUCTO}

Otra condición de sostenibilidad puede derivarse de la existencia de un límite máximo de la proporción del producto que debe ser transferida al exterior para atender los compromisos de la deuda.

Supongamos un aumento de la prima de riesgo país que resulta sostenible con el criterio de no explosividad. En el proceso que genera el aumento de la prima, el superávit comercial como proporción del producto tiende a crecer continuadamente. Consecuentemente, se reduce continuadamente la relación absorción/producto. Paralelamente, la relación deuda/producto crece hasta alcanzar un máximo en cierto momento. De allí en adelante, si los parámetros no se modifican, el superávit como proporción del producto continúa creciendo y la relación deuda/producto tiende a caer.

Puede imaginarse que una vez alcanzado el máximo de la relación deuda/ producto, la política económica procuraría instrumentar medidas para acelerar la tasa de crecimiento (y consecuentemente la tasa de aumento de las importaciones) preservando la sostenibilidad, estabilizando o reduciendo el ritmo de caída de la relación deuda/producto y estabilizando o amortiguando la contracción de la relación absorción/producto.

El proceso descrito no podría ocurrir si antes de alcanzarse la relación deuda/ producto máxima, la relación absorción/producto encontrara un límite mínimo. En este caso la deuda resulta insostenible.

El límite de contracción de la absorción está determinado esencialmente por la existencia de un mínimo admisible de la tasa de consumo de la economía, ya que la tasa de inversión tiene un piso que no podría franquearse sin afectar las tasas de crecimiento del producto y las exportaciones. ${ }^{7}$

\footnotetext{
${ }^{7}$ Este argumento estuvo en boga en la primera mitad de los años ochenta, cuando se discutía el ajuste de las economías de América Latina a las condiciones posteriores a la crisis.
} 
Podemos tratar formalmente esta condición de sostenibilidad a partir de la expresión (12):

$$
\frac{d(D / Y)}{D / Y}=\hat{D}-y=\gamma-y-\delta\left(\frac{X-M}{Y}\right) / \frac{D}{Y}
$$

Esta tasa se hace nula cuando la relación deuda/producto está en su máximo:

$$
0=\gamma-y-\delta\left(\frac{X-M}{Y}\right) /\left(\frac{D}{Y}\right)_{\text {máx }}
$$

y por lo tanto:

$$
\left(\frac{D}{Y}\right)_{\text {máx }}=\delta\left(\frac{X-M}{Y}\right) /(\gamma-y)
$$

Sea s la relación máxima admisible de la relación superávit comercial/producto (la absorción/producto mínima es entonces 1- s). Sustituyendo en (26), la condición de sostenibilidad puede escribirse:

$$
\left(\frac{D}{Y}\right)_{\operatorname{máx}} \leq \frac{\delta s}{\gamma-y}
$$

La condición (27) especifica un techo para la relación deuda/producto que puede alcanzar la economía sin transgredir el máximo de transferencia externa admisible. Para una prima de riesgo país dada, que determina una trayectoria de endeudamiento no explosiva, la deuda no resulta sostenible si la trayectoria de la relación deuda/ producto viola la expresión (27). ${ }^{8}$

Obsérvese que por la vía de especificar un flujo máximo de transferencia externa admisible se llega a determinar un límite máximo de la relación deuda/producto. La condición es formalmente análoga a la que resulta de suponer directamente que los países enfrentan una restricción de financiamiento externo (proporcional a su nivel de producto). Claro está que en (27) la restricción no es mecánica, sino que depende de la tasa de interés internacional y de la prima de riesgo país. El máximo posible de la relación deuda/producto es menor cuanto mayor es la prima de riesgo país.

En el Gráfico 6 ilustramos esta condición de sostenibildad. En el gráfico dibujamos la trayectoria de la relación deuda/producto para determinada prima de riesgo $k$. La trayectoria satisface la condición de no explosividad. Si la proporción máxima de transferencia externa admisible es $s_{1}$, la condición no es operativa y la deuda es sostenible. Con una transferencia externa máxima admisible menor $s_{2}$, la recta de restricción corta la trayectoria de la relación deuda/producto antes que ésta alcance su máximo. En este caso la deuda resulta insostenible.

\footnotetext{
${ }^{8} \mathrm{Con} \mathrm{L}=10$ años, $\mathrm{r}^{*}=5 \%, \mathrm{k}=500 \mathrm{pb}$ y una tasa máxima de transferencia externa $\mathrm{s}=3 \%$ del producto, la relación deuda/producto no podría superar el $51 \%$.
} 


\section{EQUILIBRIOS MÚLTIPLES II}

Cada una de las condiciones de sostenibilidad consideradas abre la posibilidad de equilibrios múltiples si éstas son incorporadas en la valoración del riesgo de los inversores internacionales. La posibilidad de equilibrios múltiples surge de suponer, por ejemplo, que la prima de riesgo es mayor cuanto menor es la distancia a cada una de las restricciones que imponen las condiciones discutidas. Estos puede expresarse formalmente suponiendo que la prima de riesgo está determinada por la siguiente función:

$$
k=K\left[H(k)-D_{0} / X_{0} ; y ; \frac{\delta s}{\gamma-y}-\left(\frac{D}{Y}\right)_{\text {máx }}\right] \quad K_{1}<0 ; K_{2}<0 ; K_{3}<0
$$

Con esta determinación de la prima de riesgo pueden generalizarse a cada una de las condiciones de sostenibilidad los argumentos expuestos arriba acerca de los equilibrios múltiples y las profecías autocumplidas.

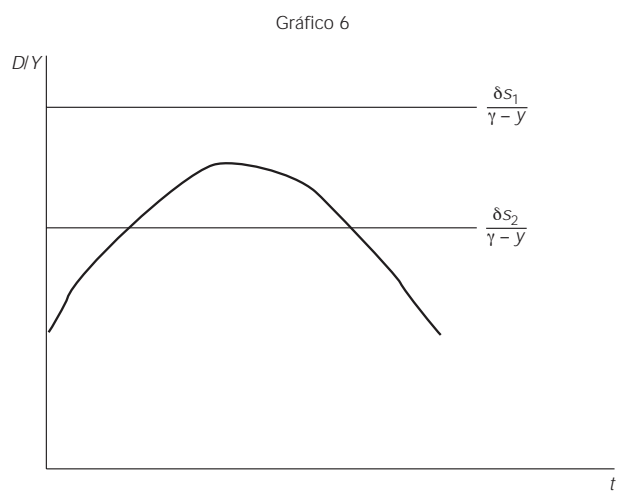

\section{ANEXO}

La condición de primer orden es la ecuación (20):

$$
\begin{aligned}
& \frac{\partial(D / Y)}{\partial t}=\frac{X_{0}}{Y_{0}}(\gamma-y) e^{(\gamma-y) t}\left(\frac{D_{0}}{X_{0}}+\frac{\delta}{x-\gamma}-\frac{\delta}{m-\gamma}\right)- \\
& \left.-\left[\frac{\delta}{x-\gamma}(x-y) e^{(x-y) t}-\frac{\delta}{m-\gamma}(m-y) e^{(m-y) t}\right]\right\}=0
\end{aligned}
$$

Como $\frac{X_{0}}{Y_{0}}>0$, es la expresión entre corchetes la que debe anularse. Dividiendo la expresión entre corchetes por $\delta(\gamma-y) e^{(\gamma-y) t}$ resulta:

$$
\frac{1}{\delta} \frac{D_{0}}{X_{0}}+\frac{1}{x-\gamma}-\frac{1}{m-\gamma}-\left[\frac{1}{x-\gamma} \frac{x-y}{\gamma-y} e^{(x-\gamma) t}-\frac{1}{m-\gamma} \frac{m-y}{\gamma-y} e^{(m-\gamma) t}\right]=0
$$


El problema es mostrar que existe un $t>0$ que satisface la ecuación (i) con los parámetros $x>m$ y $\gamma>y$.

Hacemos:

$$
A=\frac{1}{x-\gamma} \frac{x-y}{\gamma-y} \quad \text { y } \quad B=\frac{1}{m-\gamma} \frac{m-y}{\gamma-y}
$$

Con esto, la expresión (i) puede escribirse como:

$$
F(t)=A e^{(x-\gamma) t}+B e^{(m-\gamma) t}-(A-B)=\frac{1}{\delta} \frac{D_{0}}{X_{0}}
$$

donde:

$$
A-B=\frac{m-x}{(x-\gamma)(m-\gamma)}
$$

Obsérvese que $F(0)=0$ y $F^{\prime}(t)>0$.

Consideramos tres casos:

1. $x>\gamma \wedge m>\gamma$

2. $x>\gamma \wedge m<\gamma$

3. $x<\gamma(\Rightarrow m<\gamma)$

Casos 1) y 2). En estos casos $F(t) \rightarrow \infty$. Siempre existe un valor de $t>0$ tal que $F(t)=\frac{1}{\delta} \frac{D_{o}}{X_{0}}$

Caso 3). En este caso $F(t) \rightarrow-(A-B)$. Por lo tanto, solamente existe una valor de $t>0$ tal que $F(t)=\frac{1}{\delta} \frac{D_{o}}{X_{0}}$, si $\frac{1}{\delta} \frac{D_{0}}{X_{0}}<-(A-B)=\frac{x-m}{(x-\gamma)(m-\gamma)}$. Consecuentemente, la condición es:

$$
\frac{D_{o}}{X_{0}}<\frac{\delta(x-m)}{(x-\gamma)(m-\gamma)}
$$

\section{REFERENCIAS}

BACHA, Edmar (1986) "Crecimiento con oferta limitada de divisas: una revaluación del modelo de dos brechas”, en Edmar Bacha, El milagro y la crisis. Economía Brasileña y Latinoamericana, Fondo de Cultura Económica, México.

BHADURI, Amit (2001) "On the viability of external debt”, en Kazimierz Laski (ed.) External Constraints on Sustainable Growth in Transition Countries, Working Paper $\mathrm{n}^{\circ} 19$. Vienna Institute for International Economic Studies, September.

FRENKEL, Roberto (2001)"Reflexiones sobre el financiamiento del desarrollo", en Revista de la CE$P A L, \mathrm{n}^{\circ}$ 74. Santiago de Chile, agosto.

SIMONSEN, Mario Henrique (1985), "The developing-country debt problem”, en Gordon W. Smith and John T. Cuddington (eds.) International Debt and the Developing Countries. The World Bank, Washington, D.C. 